\title{
Kinematical superalgebras and Lie algebras of order 3
}

\author{
R. Campoamor-Stursberg*b and M. Rausch de Traubenberg ${ }^{\dagger b}$ \\ ${ }^{a}$ IMI and Dpto. Geometría y Topologia, Fac. CC. Matemáticas, \\ Universidad Complutense de Madrid, Plaza de Ciencias, 3, F-28040 Madrid, Spain \\ ${ }^{b}$ Laboratoire de Physique Théorique, CNRS UMR 7085, Université Louis Pasteur \\ 3 rue de l'Université, 67084 Strasbourg Cedex, France
}

\begin{abstract}
We study and classify kinematical algebras which appear in the framework of Lie superalgebras or Lie algebras of order three. All these algebras are related through generalised Inonü-Wigner contractions from either the orthosymplectic superalgebra or the de Sitter Lie algebra of order three.
\end{abstract}

\section{Introduction}

The theory of contractions of Lie groups entered physics providing a formal derivation of classical mechanics from relativistic mechanics by means of a limiting process between the underlying symmetry groups. This approach allowed a precise interpretation of the changes of physical generators during contraction, and allowed also to obtain the Poincaré group as the limit of the de Sitter groups, which constitute the only symmetry groups of General Relativity having the same dimension. Bacry and Lévy-Leblond [1] systematically applied the contractions of Lie algebras to classify the possible kinematical groups, basing on isotropy of space and the following assumptions on the structure of 4-dimensional (homogeneous) space-time: (i) Time-reversal and parity are automorphisms of the kinematical group, (ii) Non-compactness of one-parametric subgroups generated by boost transformations.

A remarkable fact on this classification is that all these kinematical models arise as contractions of the real forms of the rank two simple orthogonal Lie algebra $\mathfrak{s o}(5)$. In addition to the well known Galilean algebra, another non-relativistic kinematical group was found and studied in detail [2. This proved the versatility of contractions, allowing to explain different models in a physically consistent manner, expressing them through limits of the fundamental constants 1 The same idea of relating kinematical structures by means of contractions has been successfully applied to more general cases and situations, like superalgebras in the supersymmetric frame, or more recently quantum groups [4, 5. In these constructions, the corresponding Poincare and Galilean algebras have been derived using the supersymmetric and quantum versions of the de Sitter algebras, extending in natural manner the classical kinematical frame. Most of these works focus principally on the corresponding Poincaré and Galilei models, leaving out the analysis of generalisations of the non-standard kinematical groups like those of Carroll or Newton type, which are more or less regarded as exotic models.

A lot of attention has been also devoted to non-relativistic limits of supersymmetric theories [6], in analogy with the classical Galilean relativity studied in [7]. In [6], a systematic study of the Galilean limit of the superfield formulation of the massive Wess-Zumino model and of supersymmetric quantum electrodynamics (SQED) was developed. Criteria allowing the definition of a super-Galilei group were obtained using the notion of pseudo extension groups, emphasising the role of the mass when expanding Galilean chiral superfields. It was already pointed out there that in the massive case in higher dimensions, more than one possible limit arises from the corresponding super-Poincaré algebra, with a priori no distinguished contracted group.

\footnotetext{
*e-mail: rutwig@pdi.ucm.es

$\dagger$ e-mail: rausch@lpt1.u-strasbg.fr

${ }^{1} \mathrm{~A}$ general scheme on possible physical limit procedures can be found in [3].
} 
The main objective of this work is to extend the classical kinematical classification of Bacry and LévyLeblond to the supersymmetric case and Lie algebras of order three, using the same contraction ansatz, and including the non-standard models of Carroll and Newton. It will turn out that, with some exceptions, for the superalgebras and Lie algebras of order three, similar contraction diagrams to those found in [1] can be obtained.

Lie algebras of order three (or more generally Lie algebras of order $F$ ) were introduced in 8 as a possible generalisation of Lie (super)algebras, in order to implement non-trivial extensions of the Poincaré symmetry which are different than the usual supersymmetric extension 9 . A Lie algebra of order $F$ admits a $\mathbb{Z}_{F}$-grading $(F=3$ in this paper), the zero-graded part being a Lie algebra. An $F$-fold symmetric product (playing the role of the anticommutator in the case $F=2$ ) expresses the zero graded part in terms of the non-zero graded part. This new structure was subsequently applied within the framework of the Poincaré algebra, and a Quantum Field Theory with a non-trivial extension, different from supersymmetry, was explicitly constructed [9]. Furthermore, the basis of the theory of contractions and deformations in the context of Lie algebras of order three has been studied in [10].

The notion of contraction of Lie algebras, although generally given in terms of a representative of the isomorphism class, follows more naturally from the geometry of orbits. Given a Lie algebra $\mathfrak{g}$ with structure tensor $C_{i j}^{k}$ over a fixed basis $\left\{X_{i}\right\}, i=1, . ., n$, a linear redefinition of the generators via a matrix $A \in G L(n, \mathbb{R})$ gives the transformed structure tensor

$$
C^{\prime n}{ }_{i j}^{n}=A_{i}{ }^{k} A_{j}^{\ell}\left(A^{-1}\right)_{m}{ }^{n} C_{k \ell}^{m} .
$$

Taking into account all possible changes of basis, we obtain the orbit $\mathcal{O}(\mathfrak{g})$ of $\mathfrak{g}$ by the action of the general linear group $G L(n, \mathbb{R})$, consisting of all Lie algebras that are isomorphic to $\mathfrak{g}$. Thus, for describing the Lie algebra, any of the elements (representatives) of the orbit can be chosen. In this coordinate free interpretation, a Lie algebra $\mathfrak{g}^{\prime}$ is called a contraction of $\mathfrak{g}$ if $\mathfrak{g}^{\prime}$ belongs to the closure $\overline{\mathcal{O}(\mathfrak{g})}$ of the orbit. This naturally leads to the traditional presentation in terms of limits. Considering a family $\Phi_{\epsilon} \in \operatorname{Aut}(\mathfrak{g})$ of non-singular linear maps of $\mathfrak{g}$, where $\epsilon \in(0,1]$, for any $X, Y \in \mathfrak{g}$ we define

$$
[X, Y]_{\Phi_{\epsilon}}:=\Phi_{\epsilon}^{-1}\left[\Phi_{\epsilon}(X), \Phi_{\epsilon}(Y)\right]
$$

which obviously reproduces the brackets of the Lie algebra over the transformed basis. Actually this is nothing but equation (1.1) for a special kind of transformations. Now suppose that the limit

$$
[X, Y]_{\infty}:=\lim _{\epsilon \rightarrow 0} \Phi_{\epsilon}^{-1}\left[\Phi_{\epsilon}(X), \Phi_{\epsilon}(Y)\right]
$$

exists for any $X, Y \in \mathfrak{g}$. Then equation (1.3) defines a Lie algebra $\mathfrak{g}^{\prime}$ which is a contraction of $\mathfrak{g}$, since it corresponds to a limiting point of the orbit. We say that the contraction is non-trivial if $\mathfrak{g}$ and $\mathfrak{g}^{\prime}$ are non-isomorphic, i.e., if $\mathfrak{g}^{\prime}$ is a point of the frontier of $\mathcal{O}(\mathfrak{g})$, and trivial otherwise. In this sense contractions should be understood as points of paths connecting two representatives of the orbits of the corresponding Lie algebras. As a consequence, two contractions $\mathfrak{g}_{1} \stackrel{f_{\epsilon}}{\longrightarrow} \mathfrak{g}_{2}$ and $\mathfrak{g}_{1}^{\prime} \stackrel{g_{\epsilon}}{\longrightarrow} \mathfrak{g}_{2}^{\prime}$ are equivalent if $\mathfrak{g}_{1}^{\prime} \in \mathcal{O}\left(\mathfrak{g}_{1}\right)$ and $\mathfrak{g}_{2}^{\prime} \in \mathcal{O}\left(\mathfrak{g}_{2}\right)$.

A contraction for which there exists some basis $\left\{Y_{1}, . ., Y_{n}\right\}$ 这 such that the contraction matrix $A_{\Phi}$ is diagonal, that is, adopts the form

$$
\left(A_{\Phi}\right)_{i j}=\delta_{i j} \epsilon^{n_{j}}, \quad n_{j} \in \mathbb{Z},
$$

is called a generalised Inönü-Wigner contraction. Although there exist contractions that are not equivalent to this type, which means that an arbitrary contraction does not necessarily reduce to this form, for many physical problems they are of great relevance. The diagonal transformations correspond to some scale changes in the generators, and therefore have a precise physical meaning. In was precisely in this sense that contractions were introduced by Segal, Inönü and Wigner [11, in order to describe a continuous transition from relativistic to non-relativistic physics. These definitions can be generalised without effort to superalgebras and other more general algebraic structures [12].

\footnotetext{
${ }^{2}$ The choice of basis determines the structure constants, and therefore an orbit representative of $\mathcal{O}(\mathfrak{g})$
} 
In this work we focus primarily on generalised Inönü-Wigner (IW) contractions, for physical reasons. Since in the kinematical frame the generators of the Lie algebra are identified with physical operators, contractions obtained by re-scaling certain of its generators still preserve this physical meaning, up to some phase transitions for the rescaled elements. On the other hand, the class of IW-contractions is the natural type of limiting transformations related to semisimple Lie algebras and the embedding of subalgebras [13]. Even if successive composition of IW-contractions is not necessarily equivalent to a general IW-contraction, in each step we deal with diagonal transformations, which enables us to interpret how the symmetry changes when modifying the main parameters.

The content of this paper is the following: In section two we review the supersymmetric and Lie algebras of order three extensions of the de Sitter algebra. Section three (respectively four, five and six) are devoted to the study of extensions of the Poincaré algebra (respectively to the extensions of the Galilei algebra, of the Carroll algebra and of the Newton algebra). Although there is only one physically consistent possible supersymmetric extension of the corresponding Lie algebras, there are many possible extensions of order three. Some of them reproduce already known extensions considered in the literature. It turns out that, in analogy to the Lie algebra case, the kinematical superalgebras (resp. kinematical Lie algebras of order three) are related though an IW-contraction of the orthosymplectic superalgebra (resp. de Sitter algebra of order three). Finally, more extended material related to the algebraic definition of a Lie algebra of order three is given in the appendix.

\section{Extensions of the (anti) de Sitter algebras}

We consider in this section some possible extensions of the de Sitter Lie algebras $\mathfrak{s o}(2,3)$ and $\mathfrak{s o}(1,4)$. Since the difference between these two models is the signature of the metric tensor, the expressions involving the generators can be developed without particular reference to the metric. In the following, we just give the algebraic structure for the anti-de Sitter case $\mathfrak{s o}(2,3)$, the argument being similar for the remaining model by simply replacing the metric tensor. We consider the usual basis $\left\langle L_{M N}=-L_{N M}, 0 \leq M<N \leq 4\right\rangle$ with commutation relations

$$
\left[L_{M N}, L_{P Q}\right]=\eta_{N P} L_{M Q}-\eta_{M P} L_{N Q}+\eta_{Q N} L_{P M}-\eta_{Q M} L_{P N}
$$

where $\eta_{M N}=\operatorname{diag}(1,-1,-1,-1,1)$. It follows at once that the generators $L_{M N}$ with $M, N \neq 4$ span the Lorentz algebra $\mathfrak{s o}(1,3)$. In the basis $<L_{\mu \nu}, P_{\mu}=L_{\mu 4}, \mu, \nu=0, \cdots, 3>$ the commutation relations are rewritten as

$$
\begin{aligned}
{\left[L_{\mu \nu}, L_{\rho \sigma}\right] } & =\eta_{\nu \rho} L_{\mu \sigma}-\eta_{\mu \rho} L_{\nu \sigma}+\eta_{\sigma \nu} L_{\rho \mu}-\eta_{\sigma \mu} L_{\rho \nu} \\
{\left[L_{\mu \nu}, P_{\rho}\right] } & =\eta_{\nu \rho} P_{\mu}-\eta_{\mu \rho} P_{\nu} \\
{\left[P_{\mu}, P_{\nu}\right] } & =L_{\nu \mu} .
\end{aligned}
$$

For later use, when dealing with contractions of $\mathfrak{s o}(2,3)$, we also rewrite this algebra in the Bacry-LévyLeblond notation: We rename the basis elements as $K_{i}=L_{0 i}, P_{i}=L_{i 4}, H=L_{04}$ and $L_{i}=L_{j k}$, where $i, j, k$ are taken in cyclic order. From now on, any relation like $\left[L_{i}, L_{j}\right]=L_{k}$ means that $i, j, k$ are taken in cyclic order. The brackets of the anti-de Sitter algebra over this basis are:

$$
\begin{array}{llll}
{\left[L_{i}, L_{j}\right]=L_{k},} & {\left[L_{i}, K_{j}\right]=K_{k},} & {\left[L_{i}, P_{j}\right]=P_{k},} & {\left[K_{i}, K_{j}\right]=-L_{k},} \\
{\left[P_{i}, P_{j}\right]=-L_{k},} & {\left[K_{i}, P_{j}\right]=-\delta_{i j} H,} & {\left[K_{i}, H\right]=-P_{i},} & {\left[P_{i}, H\right]=K_{i} .}
\end{array}
$$

In order to shorten notations, we will write $[\mathbf{L}, \mathbf{L}]=\mathbf{L}$ for $\left[L_{i}, L_{j}\right]=L_{k}$. As proved in [1, all classical kinematical algebras arise as Inönü-Wigner contractions of the de Sitter algebras. The brackets of the contractions are reproduced in Table 1.

It is straightforward to verify that the Poincaré and Para-Poincaré algebras $\left(I \mathfrak{s o}(1,3)\right.$ and $I \mathfrak{s o}(1,3)^{\prime}$, respectively) are isomorphic as Lie algebras, though they are physically different. The same happens with the two Galilei algebras. The physical distinction follows from the interpretation of the generators. For this reason, from now on, we will only consider the Poincaré and Galilei algebras, the computations being completely analogous for the Para-models by simply interchanging the generators. 
Table 1: Non-vanishing brackets of classical kinematical algebras in the standard basis. The common brackets to all Lie algebras below are those corresponding to space isotropy: $[\mathbf{L}, \mathbf{L}]=\mathbf{L},[\mathbf{L}, \mathbf{K}]=\mathbf{K}$ and $[\mathbf{L}, \mathbf{P}]=\mathbf{P}$.

\begin{tabular}{c|cccccccccc}
\hline \hline & $\mathfrak{s o}(2,3)$ & $\mathfrak{s o}(1,4)$ & $I \mathfrak{s o}(1,3)$ & $I \mathfrak{s o}(4)$ & $I \mathfrak{s o}(1,3)^{\prime}$ & Carroll & $N e^{\exp }$ & $N e^{\text {osc }}$ & $G(2)$ & $G(2)^{\prime}$ \\
\hline$[\mathbf{K}, \mathbf{K}]$ & $-\mathbf{L}$ & $-\mathbf{L}$ & $-\mathbf{L}$ & 0 & 0 & 0 & 0 & 0 & 0 & 0 \\
{$[\mathbf{K}, \mathbf{P}]$} & $-H$ & $-H$ & $-H$ & $-H$ & $-H$ & $-H$ & 0 & 0 & 0 & 0 \\
{$[\mathbf{P}, \mathbf{P}]$} & $-\mathbf{L}$ & $\mathbf{L}$ & 0 & $\mathbf{L}$ & $-\mathbf{L}$ & 0 & 0 & 0 & 0 & 0 \\
{$[\mathbf{K}, H]$} & $-\mathbf{P}$ & $-\mathbf{P}$ & $-\mathbf{P}$ & 0 & 0 & 0 & $-\mathbf{P}$ & $-\mathbf{P}$ & $-\mathbf{P}$ & 0 \\
{$[\mathbf{P}, H]$} & $\mathbf{K}$ & $-\mathbf{K}$ & 0 & $-\mathbf{K}$ & $\mathbf{K}$ & 0 & $-\mathbf{K}$ & $\mathbf{K}$ & 0 & $-\mathbf{K}$ \\
\hline \hline
\end{tabular}

\subsection{The $\mathfrak{o s p}(1 \mid 4)$ algebra}

To construct a supersymmetric extension of the (anti-)de Sitter algebra, we start from the real forms of the orthosymplectic superalgebra $\mathfrak{o s p}(1 \mid 4, \mathbb{C})=\mathfrak{s p}(4, \mathbb{C}) \oplus \mathbb{C}^{4}$. Among the Lie algebras $\mathfrak{s o}(2,3)$ and $\mathfrak{s o}(1,4)$, only the former admits a four-dimensional real spinor representation (the Majorana spinors ), therefore only $\mathfrak{s o}(2,3)$ will admit a supersymmetric extension. Using the natural inclusion $\mathfrak{s o}(1,3) \subset \mathfrak{s o}(2,3)$ in the basis (2.1), we consider the following decomposition $\mathfrak{o s p}(1 \mid 4)=\mathfrak{s o}(2,3) \oplus \mathbb{R}^{4}=\left\langle L_{\mu \nu}, P_{\mu}\right\rangle \oplus\left\langle S_{\alpha}, \bar{S}^{\dot{\alpha}}, \alpha, \dot{\alpha}=1,2\right\rangle$, where $\left(S_{\alpha}, \bar{S}^{\dot{\alpha}}\right)$ is a four dimensional Majorana spinor $\left(\bar{S}^{\dot{\alpha} \star}=S^{\alpha}\right.$, where ${ }^{\star}$ denotes the complex conjugation) of $\mathfrak{s o}(1,3)$. Now, taking for the Dirac $\Gamma$ - matrices

$$
\Gamma_{M}=\left\{\begin{array}{l}
\Gamma_{\mu}=\left(\begin{array}{cc}
0 & \sigma_{\mu} \\
\bar{\sigma}_{\mu} & 0
\end{array}\right) \\
\Gamma_{4}=\left(\begin{array}{cc}
1 & 0 \\
0 & -1
\end{array}\right)
\end{array}\right.
$$

with $\sigma_{\mu}=\left(1, \sigma_{i}\right), \bar{\sigma}_{\mu}=\left(1,-\sigma_{i}\right)$, the $\sigma_{i}(i=1,2,3)$ denoting the Pauli spin matrices. The index structure of the $\sigma_{\mu}$-matrices is as follows: $\sigma_{\mu} \rightarrow \sigma_{\mu \alpha \dot{\alpha}}, \bar{\sigma}_{\mu} \rightarrow \bar{\sigma}_{\mu}^{\dot{\alpha} \alpha}$. We also define $S_{\alpha}=\varepsilon_{\alpha \beta} S^{\beta}, S^{\alpha}=\varepsilon^{\alpha \beta} S_{\beta}$, $\bar{S}_{\dot{\alpha}}=\bar{\varepsilon}_{\dot{\alpha} \dot{\beta}} \bar{S}^{\dot{\beta}}, \bar{S}^{\dot{\alpha}}=\bar{\varepsilon}^{\dot{\alpha} \dot{\beta}} \bar{S}_{\dot{\beta}}$ with $\varepsilon, \bar{\varepsilon}$ antisymmetric matrices given by $\varepsilon_{12}=\bar{\varepsilon}_{\dot{1} \dot{2}}=-1$ and $\varepsilon^{12}=\bar{\varepsilon}^{-\dot{i} \dot{ }}=1$, respectively. This means that if we define

$$
S_{A}=\left(\begin{array}{c}
S_{\alpha} \\
\bar{S}^{\dot{\alpha}}
\end{array}\right)
$$

a Majorana spinor of $\mathfrak{s o}(1,3)$, we have that

$$
S^{A}=C_{4}^{A B} S_{B}, \quad C_{4}^{A B}=\left(\begin{array}{cc}
\epsilon^{\alpha \beta} & 0 \\
0 & \bar{\epsilon}_{\dot{\alpha} \dot{\beta}}
\end{array}\right),
$$

and $C_{4}$ defines a metric on the spinor space, seen as a $(1+3) D$-spinor (see e.g. [14). Introducing now the $\mathfrak{s o}(2,3)$ generators in the spinor representation

$$
\Gamma_{M N}=\frac{1}{4}\left(\Gamma_{M} \Gamma_{N}-\Gamma_{N} \Gamma_{M}\right)=\left\{\begin{array}{l}
\Gamma_{\mu \nu}=\left(\begin{array}{cc}
\sigma_{\mu \nu} & 0 \\
0 & \bar{\sigma}_{\mu \nu}
\end{array}\right)=\left(\begin{array}{cc}
\frac{1}{4}\left(\sigma_{\mu} \bar{\sigma}_{\nu}-\sigma_{\nu} \bar{\sigma}_{\mu}\right) & 0 \\
0 & -\frac{1}{2} \sigma_{\mu} \\
\Gamma_{\mu 4} & =
\end{array}\right) \\
\frac{1}{2} \bar{\sigma}_{\mu}\left(\bar{\sigma}_{\mu} \sigma_{\nu}-\bar{\sigma}_{\nu} \sigma_{\mu}\right)
\end{array}\right)
$$

the index structure of the $\sigma_{\mu \nu}$-matrices is reformulated as $\sigma_{\mu \nu} \rightarrow\left(\sigma_{\mu \nu}\right)_{\alpha}{ }^{\beta}, \bar{\sigma}_{\mu \nu} \rightarrow\left(\bar{\sigma}_{\mu \nu}\right)^{\dot{\alpha}}{ }_{\dot{\beta}}$. To construct the orthosymplectic superalgebra $\mathfrak{o s p}(1 \mid 4)$, we notice at first that if we consider now $S_{A}$ as an $\mathfrak{s o}(2,3)$ Majorana spinor, the spinor metric is given by

$$
C_{5}^{A B}=\left(\begin{array}{cc}
\epsilon^{\alpha \beta} & 0 \\
0 & -\bar{\epsilon}_{\dot{\alpha} \dot{\beta}}
\end{array}\right),
$$

and, with respect to $C_{5} \Gamma_{M N A}{ }^{B} C_{5 D B}$, are symmetric matrices (see for instance [14]). Now, since our conventions are such that the R.H.S. of all commutators for a Lie algebra have no pure imaginary factor $i$ (i.e. the structure constants are real), for an unitary representation the generators of the algebra are given 
by antihermitian operators (this convention is different with respect to that usually considered in physics). This means that in order to construct the orthosymplectic algebra with real structure constants, since in the Majorana representation the $\Gamma_{M N}$ matrices are real, we need to consider generators in the fermionic sector which respect this convention: we will use rescaled $S_{A}$ 's such that $S_{\alpha}^{\star}=i \bar{S}_{\dot{\alpha}}$ and $\bar{S}_{\dot{\alpha}}^{\star}=i S_{\alpha}$. With this notation, the $\mathfrak{o s p}(1 \mid 4)$ algebra read: 3

$$
\left\{S_{A}, S_{B}\right\}=b \Gamma_{M N A}{ }^{D} C_{5 B D} L^{M N},
$$

with $b$ positive or $b$ negative. The two choices for the sign of $b$ correspond to the two real forms of $\mathfrak{o s p}(1 \mid 4, \mathbb{C})$. In the $\mathfrak{s o}(1,3)$ notations, the algebra takes the form (we have chosen here the real form corresponding to $b>0$ and we have normalised the generators such that $b=4)$

$$
\begin{aligned}
& {\left[L_{\mu \nu}, S_{\alpha}\right]=\left(\sigma_{\mu \nu}\right)_{\alpha}{ }^{\beta} S_{\beta}, \quad\left[L_{\mu 4} S_{\alpha}\right]=-\frac{1}{2} \sigma_{\mu \alpha \dot{\alpha}} \bar{S}^{\dot{\alpha}}, \quad\left[L_{\mu \nu}, \bar{S}^{\dot{\alpha}}\right]=\left(\bar{\sigma}_{\mu \nu}\right)^{\dot{\alpha}} \bar{S}^{\dot{\beta}}, \quad\left[L_{\mu 4}, \bar{S}^{\dot{\alpha}}\right]=\frac{1}{2} \bar{\sigma}_{\mu}^{\dot{\alpha} \alpha} S_{\alpha},} \\
& \left\{S_{\alpha}, S_{\beta}\right\}=4\left(\sigma^{\mu \nu}\right)_{\alpha \beta} L_{\mu \nu},\left\{\bar{S}^{\dot{\alpha}}, \bar{S}^{\dot{\beta}}\right\}=-4\left(\bar{\sigma}^{\mu \nu}\right)^{\dot{\alpha} \dot{\beta}} L_{\mu \nu},\left\{S_{\alpha}, \bar{S}_{\dot{\beta}}\right\}=2\left(\sigma^{\mu}\right)_{\alpha \dot{\beta}} P_{\mu} .
\end{aligned}
$$

together with those of (2.2).

\subsection{The de Sitter algebra of order 3}

With the previous notations, we are in situation of constructing an anti-de Sitter algebra of order 3. (See the Appendix for the definition of Lie algebras of order three.) To this extent, we consider the following Lie algebra decomposition: $\mathfrak{g}=\mathfrak{s o}(2,3) \oplus$ ad $\mathfrak{s o}(2,3)=\left\langle L_{M N}=-L_{N M}, 0 \leq M<N \leq 4\right\rangle \oplus$ $\left\langle A_{M N}=-A_{N M}, 0 \leq M<N \leq 4 \sqrt{4}\right.$ with additional bilinear and trilinear brackets:

$$
\begin{aligned}
{\left[L_{M N}, A_{P Q}\right] } & =\eta_{N P} A_{M Q}-\eta_{M P} A_{N Q}+\eta_{Q N} A_{P M}-\eta_{Q M} A_{P N} \\
\left\{A_{M N}, A_{P Q}, A_{R S}\right\} & =\left(\eta_{M P} \eta_{N Q}-\eta_{M Q} \eta_{N P}\right) L_{R S}+\left(\eta_{M R} \eta_{N S}-\eta_{M S} \eta_{N R}\right) L_{P Q}+\left(\eta_{P R} \eta_{Q S}-\eta_{P S} \eta_{Q R}\right) L_{M N}
\end{aligned}
$$

together with the relations (2.1). Using the $\mathfrak{s o}(1,3)$-basis previously mentioned, ad $\mathfrak{s o}(2,3)$ is generated by $A_{\mu \nu}, C_{\mu}=A_{\mu 4}, \mu, \nu=0,1,2,3$, and the algebra reads

$$
\begin{aligned}
{\left[L_{\mu \nu}, A_{\rho \sigma}\right] } & =\eta_{\nu \rho} A_{\mu \sigma}-\eta_{\mu \rho} A_{\nu \sigma}+\eta_{\sigma \nu} A_{\rho \mu}-\eta_{\sigma \mu} A_{\rho \nu}, \\
{\left[L_{\mu \nu}, C_{\rho}\right] } & =\eta_{\nu \rho} C_{\mu}-\eta_{\mu \rho} C_{\nu}, \\
{\left[P_{\mu}, A_{\nu \rho}\right] } & =-\eta_{\rho \mu} C_{\nu}+\eta_{\nu \mu} C_{\rho}, \\
{\left[P_{\mu}, C_{\nu}\right] } & =A_{\nu \mu} . \\
\left\{A_{\mu \nu}, A_{\pi \kappa}, A_{\rho \sigma}\right\} & =\left(\eta_{\mu \pi} \eta_{\nu \kappa}-\eta_{\mu \kappa} \eta_{\nu \pi}\right) L_{\rho \sigma}+\left(\eta_{\mu \rho} \eta_{\nu \sigma}-\eta_{\mu \sigma} \eta_{\nu \rho}\right) L_{\pi \kappa}+\left(\eta_{\pi \rho} \eta_{\kappa \sigma}-\eta_{\pi \sigma} \eta_{\kappa \rho}\right) L_{\mu \nu}, \\
\left\{A_{\mu \nu}, A_{\pi \kappa}, C_{\rho}\right\} & =\left(\eta_{\mu \pi} \eta_{\nu \kappa}-\eta_{\mu \kappa} \eta_{\nu \pi}\right) P_{\rho}, \\
\left\{A_{\mu \nu}, C_{\rho}, C_{\sigma}\right\} & =\eta_{\rho \sigma} L_{\mu \nu}, \\
\left\{C_{\mu}, C_{\nu}, C_{\rho}\right\} & =\eta_{\mu \nu} P_{\mu}+\eta_{\mu \rho} P_{\nu}+\eta_{\nu \rho} P_{\mu},
\end{aligned}
$$

together with the relations (2.2). This algebra was first introduced in [8, where it has been established that to any Lie (super)algebra we can associate a Lie algebra of order $F$ structure by means of an induction based argument. The algebra (2.4) appears as a special case of this induction theorem.

\footnotetext{
${ }^{3}$ In the convention of [15] the generators of the bosonic and fermionic part of the algebra are hermitian and thus the structure constants for all the commutators (resp. anticommutators) are purely imaginary (resp. real) although in the conventions of [16] the generators of the bosonic (resp. fermionic) part of the algebra are anti-hermitian (resp. hermitian) hence the structure constant for all the commutators (resp. anticommutators) are real (resp. purely imaginary). With our conventions, the structure constant of both the commutators and anticommutators are real.

${ }^{4}$ We could have considered, on an equal footing, the Lie algebra of order $3 \mathfrak{g}=\mathfrak{s o}(1,4) \oplus$ ad $\mathfrak{s o}(1,4)$.
} 


\section{Extensions of the Poincaré algebra}

In the classical frame, the Poincaré algebra is obtained from the anti-de Sitter algebra through the InonüWigner contraction defined by the transformations

$$
L_{\mu \nu}^{\prime}=L_{\mu \nu}, \quad P_{\mu}^{\prime}=\varepsilon P_{\mu}
$$

and taking the limit $\varepsilon \rightarrow 0$. The non-vanishing brackets are

$$
\begin{aligned}
{\left[L_{\mu \nu}^{\prime}, L_{\rho \sigma}^{\prime}\right] } & =\eta_{\nu \rho} L_{\mu \sigma}^{\prime}-\eta_{\mu \rho} L_{\nu \sigma}^{\prime}+\eta_{\sigma \nu} L_{\rho \mu}^{\prime}-\eta_{\sigma \mu} L_{\rho \nu}^{\prime} \\
{\left[L_{\mu \nu}^{\prime}, P_{\rho}^{\prime}\right] } & =\eta_{\nu \rho} P_{\mu}^{\prime}-\eta_{\mu \rho} P_{\nu}^{\prime} \\
{\left[P_{\mu}^{\prime}, P_{\nu}^{\prime}\right] } & =0
\end{aligned}
$$

It is natural to ask whether a similar procedure holds for the supersymmetric extensions. Due to isomorphism of the anti-de Sitter algebra with the non-compact symplectic algebra $\mathfrak{s p}(4, \mathbb{R})$, the correct choice for the contraction in the supersymmetric case is the orthosymplectic algebra osp $(1 \mid 4)$ instead of the superalgebras $\mathfrak{o r p}(5 \mid N) 5$ [17.

In addition to the generators of (3.1), we consider also transformed generators in the Fermi sector of $\mathfrak{o s p}(1 \mid 4)$ defined by

$$
Q_{\alpha}=\varepsilon^{a} S_{\alpha}, \quad \bar{Q}^{\dot{\alpha}}=\varepsilon^{a} \bar{S}^{\dot{\alpha}} .
$$

This contraction pattern is consistent, i.e., the limit exists for $\varepsilon \rightarrow 0$ and defines a superalgebra, if the condition $2 a \geq 1$ is satisfied. For the value $a=\frac{1}{2}$ we find the algebra

$$
\begin{aligned}
{\left[L_{\mu \nu}^{\prime} Q_{\alpha}\right] } & =\left(\sigma_{\mu \nu}\right)_{\alpha}{ }^{\beta} Q_{\beta}, \quad\left[P_{\mu}^{\prime}, Q_{\alpha}\right]=0, \\
{\left[L_{\mu \nu}^{\prime} \bar{Q}^{\dot{\alpha}}\right] } & =\left(\bar{\sigma}_{\mu \nu}\right)^{\dot{\alpha}}{ }_{\dot{\beta}} \bar{Q}^{\dot{\beta}}, \quad\left[P_{\mu}^{\prime}, \bar{Q}^{\dot{\alpha}}\right]=0, \\
\left\{Q_{\alpha}, Q_{\beta}\right\} & =0, \quad\left\{\bar{Q}^{\dot{\alpha}}, \bar{Q}^{\dot{\beta}}\right\}=0, \quad\left\{Q_{\alpha}, \bar{Q}_{\dot{\beta}}\right\}=2 \sigma^{\mu}{ }_{\alpha \dot{\beta}} P_{\mu}^{\prime},
\end{aligned}
$$

which is the well-known supersymmetric algebra. In the kinematical notations of Table 1, these brackets reduce to

$$
\begin{aligned}
& {\left[L_{k}^{\prime}, Q_{\alpha}\right]=-\frac{i}{2}\left(\sigma_{k}\right)_{\alpha}{ }^{\beta} Q_{\beta},\left[K_{k}^{\prime}, Q_{\alpha}\right]=-\frac{1}{2}\left(\sigma_{k}\right)_{\alpha}{ }^{\beta} Q_{\beta},} \\
& {\left[L_{k}^{\prime}, \bar{Q}^{\dot{\alpha}}\right]=-\frac{i}{2}\left(\sigma_{k}\right)^{\dot{\alpha}}{ }_{\dot{\beta}} \bar{Q}^{\dot{\beta}},\left[K_{k}^{\prime}, \bar{Q}^{\dot{\alpha}}\right]=\frac{1}{2}\left(\sigma_{k}\right)^{\dot{\alpha}}{ }_{\dot{\beta}} \bar{Q}^{\dot{\beta}},} \\
& \left\{Q_{\alpha}, \bar{Q}_{\dot{\beta}}\right\}=2 \sigma_{i \alpha \dot{\alpha}} P_{i}^{\prime}+2 \sigma_{0_{\alpha \dot{\beta}}} H^{\prime} .
\end{aligned}
$$

Here we have $\sigma_{i \alpha \dot{\alpha}} \bar{\sigma}_{j} \dot{\alpha} \beta=-i\left(\sigma_{k}\right)_{\alpha}{ }^{\beta}, \bar{\sigma}_{i}^{\dot{\alpha} \alpha} \sigma_{j \dot{\beta}}=-i\left(\sigma_{k}\right)_{\dot{\beta}}^{\dot{\alpha}}$ (with $i, j, k$ in circular permutation) or $\left(\sigma_{i}\right)_{\alpha}{ }^{\beta}=$ $-\sigma_{0 \alpha \dot{\alpha}} \bar{\sigma}_{i}^{\dot{\alpha} \beta}$ and $\left(\sigma_{i}\right)_{\dot{\beta}}^{\dot{\alpha}}=\bar{\sigma}_{0}{ }^{\dot{\alpha} \alpha} \sigma_{i_{\alpha \dot{\beta}}}$.

This supersymmetric extension of the Poincaré algebra was first proposed by Gol'fand and Likhtman in 1971, although its formal introduction in physics is due to Wess and Zumino [18. This algebra moreover arises as a contraction of the orthosymplectic superalgebra $\mathfrak{o s p}(1 \mid 4)$, thus leads to a consistent contraction pattern for supersymmetric extensions of kinematical algebras.

There is however another possibility of contracting the $\mathfrak{o s p}(1 \mid 4)$ superalgebra such that it is still consistent with the Poincaré algebra. This alternative was introduced in [19], and proven to be an extension of the Poincaré algebra by odd twistorial generators. Since for this extension no hermitian representations for the generators in the Fermi part exist, the so-called Konopel'chenko algebra is of no use in supersymmetry 6 Because of the non-reality of the latter algebra, we keep it out from our construction of Lie algebras of order three.

\footnotetext{
${ }^{5}$ These algebras violate the physically supersymmetric principle of requiring that the elements of the Fermi sector transform as Lorentz spinors.

${ }^{6}$ Formally both Lagrangian are quite similar, but the pure complex character for the Konopel'chenko extension prevents it from providing physically realistic descriptions.
} 


\subsection{Cubic Poincaré algebras}

Following the previous pattern of obtaining a supersymmetric extension of the Poincaré algebra by means of generalisations of the classical kinematical and supersymmetric contraction, we search for cubic extensions of the Poincaré algebra that can be obtained contracting the corresponding anti de Sitter algebra of order three. To this extent, in addition to (3.1) we consider the following re-scaled generators:

$$
U_{\mu \nu}=\varepsilon^{a} A_{\mu \nu}, \quad V_{\mu}=\varepsilon^{b} C_{\mu} .
$$

Expressing the binary and ternary brackets in this basis and taking the limit $\varepsilon \rightarrow 0$, we find that this contraction pattern is consistent only if the following system on $a, b$ is satisfied 7 :

$$
1+a-b \geq 0,1+b-a \geq 0,3 a \geq 0,2 a+b-1 \geq 0, a+2 b \geq 0,3 b-1 \geq 0 .
$$

We observe that, if all inequalities are strict, then all involved brackets vanish and the corresponding extension is trivial. In order that a bilinear or trilinear is preserved, it is necessary that the corresponding inequality on the contraction parameters is an equality. Therefore, the non-trivial extensions are given by those solutions of the preceding system for which at least one equality is satisfied. The resulting extensions with non-vanishing trilinear bracket are given schematically in Table 2 .

Table 2: Poincaré algebras of order three

\begin{tabular}{|c||l|c|c|c|c|c|c|}
\hline & & {$\left[P^{\prime}, U\right]$} & {$\left[P^{\prime}, V\right]$} & $\{U, U, U\}$ & $\{U, U, V\}$ & $\{U, V, V\}$ & $\{V, V, V\}$ \\
\hline 1. & $a=0, b=1$ & $V$ & 0 & $L$ & $P$ & 0 & 0 \\
\hline 2. & $1+a-b=0$ & $V$ & 0 & 0 & 0 & 0 & 0 \\
\hline 3. & $a=\frac{4}{3}, b=\frac{1}{3}$ & 0 & $U$ & 0 & 0 & 0 & $P$ \\
\hline 4. & $1+b-a=0$ & 0 & $U$ & 0 & 0 & 0 & 0 \\
\hline 5. & $2 a+b-1=0$ & 0 & 0 & 0 & $P$ & 0 & 0 \\
\hline 6. & $a=b=\frac{1}{3}$ & 0 & 0 & 0 & $P$ & 0 & $P$ \\
\hline 7. & 0 & 0 & 0 & 0 & 0 & $P$ \\
\hline
\end{tabular}

The precise form of the brackets can be read off from (3.6), e.g. $\{V, V, V\}=P^{\prime}$ means that $\left\{V_{\mu}, V_{\nu}, V_{\rho}\right\}=$ $\eta_{\mu \nu} P_{\rho}^{\prime}+\eta_{\mu \rho} P_{\nu}^{\prime}+\eta_{\nu \rho} P_{\mu}^{\prime}$, and the brackets $\left[L^{\prime}, U\right]$ and $\left[L^{\prime}, V\right]$ are always different from zero. The extensions where $\left[P^{\prime}, U\right]$ and/or $\left[P^{\prime}, V\right]$ are different from zero are interesting since the translations are represented by non-vanishing matrices. These are special cases of Lemma 1 of 10 . Furthermore, there is only one cubic algebra which admits the subalgebra generated by $\left\langle L_{\mu, \nu}^{\prime}, P_{\mu}^{\prime}\right\rangle \oplus\left\langle V_{\mu}\right\rangle$, which corresponds to the cubic extension studied in [9]. This cubic extension of the Poincaré algebra has been implemented in Quantum Field Theory [9]. Expressed in terms of the kinematical basis $\left\langle L_{i}^{\prime}, K_{i}^{\prime}, P_{i}^{\prime}, H^{\prime}\right\rangle \oplus\left\langle V_{i}, W=V_{0}\right\rangle$, it adopts the form

$$
\begin{array}{ll}
{\left[L_{i}^{\prime}, V_{j}\right]=V_{k},} & {\left[K_{i}^{\prime}, V_{j}\right]=-\delta_{i j} W,} \\
{\left[L_{i}^{\prime}, W\right]=0,} & {\left[K_{i}^{\prime}, W\right]=-V_{i}} \\
\left\{V_{i}, V_{j}, V_{k}\right\}=-\delta_{i j} P_{k}^{\prime}-\delta_{i k} P_{j}^{\prime}-\delta_{j k} P_{i}^{\prime} & \left\{V_{i}, V_{j}, W\right\}=-\delta_{i j} H^{\prime}, \\
\left\{V_{i}, W, W\right\}=P_{i}^{\prime}, & \{W, W, W\}=3 H^{\prime}
\end{array}
$$

In the contraction scheme above, the generators before contraction are denoted by unprimed symbols, while those after contraction are primed. From now on, for any contractions of the Poincare algebra, the generators of $I \mathfrak{s o}(1,3)$ will be unprimed while those of the contracted algebra will be primed. Since the Carroll and the Galilei algebras are obtained through an Inonü-Wigner contraction from the Poincaré algebra, we will only consider the Galilei and Carroll algebras of order three related to the Poincaré algebra of order

\footnotetext{
${ }^{7}$ By consistent contraction pattern we mean that the corresponding limit (1.3) exists for any pair and triple of generators.
} 
three (3.8) i.e. generated by $\left\langle L_{i}, K_{i}, P_{i}, H>\oplus<V_{i}, W>\right.$. This choice is justified by the interest of the algebra (3.8) in the quantum field theoretic approach, while for the remaining cubic extensions (algebras 1-6 of Table 2) no consistent model has been developed yet, thus not allowing to interpret in some physical manner the resulting contractions.

\section{Extensions of the Galilei algebra}

The Galilei algebra is obtained from the Poincaré algebra $I \mathfrak{s o}(1,3)$ through the contraction defined by the transformations

$$
L_{i}^{\prime}=L_{i}, \quad K_{i}^{\prime}=\varepsilon K_{i}, \quad P_{i}^{\prime}=\varepsilon P_{i}, \quad H^{\prime}=H
$$

The brackets are given in Table 1. In order to construct a super-Galilei algebra by means of contractions, we add to the previous generators the following ones:

$$
Q_{\alpha}^{\prime}=\epsilon^{a} Q_{\alpha}, \quad \bar{Q}_{\alpha}^{\prime}=\epsilon^{b} \bar{Q}_{\alpha}
$$

In the inclusion $\mathfrak{s o}(3) \subset \mathfrak{s o}(1,3)$, the two representations $\left\langle Q_{\alpha}\right\rangle$ and $\left\langle\bar{Q}_{\dot{\alpha}}\right\rangle$ become equivalent (but complex conjugate), we thus denote them as $\bar{Q}_{\dot{\alpha}} \rightarrow \bar{Q}_{\alpha}$ and $\sigma_{i \alpha \dot{\beta}} \rightarrow \sigma_{i \alpha \beta}$. The contraction scheme above leads to a superalgebra whenever the condition $a+b-1 \geq 0$ is satisfied. For the special case $a+b-1=0$ we get the $N=2$ supersymmetric extension (without central charge) of the Galilei algebra given by:

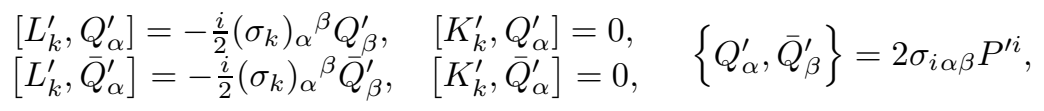

It should be remarked that, like happens with the Poincaré case, this is not the only possibility for a supersymmetric Galilei algebra. There is another possible contraction taking into account a special $\mathbb{Z}_{4^{-}}$ grading of the Poincaré superalgebra [5]. The authors are however not aware of supersymmetric field models based on this second Galilei superalgebra, which justifies that it is not considered further to obtain the corresponding Lie algebras of order three.

\subsection{Galilei algebras of order 3}

To construct the Galilei algebras of order three from the cubic extension of Poincaré (3.8), we consider the following generators

$$
V_{i}^{\prime}=\epsilon^{a} V_{i}, \quad W^{\prime}=\epsilon^{b} W
$$

in addition to those of (4.1). The constraints to be satisfied by the powers $a, b$ to define consistent contractions are (see (3.8)):

$$
1+a-b \geq 0,1+b-a \geq 0, a \geq \frac{1}{3}, 2 a+b \geq 0, a+2 b-1 \geq 0, b \geq 0 .
$$

The solutions to these equations provide the possible non-equivalent cubic extensions of the Galilei algebra. In any case, we have the common bracket $\left\{V^{\prime}, V^{\prime}, W^{\prime}\right\}=0$. The remaining brackets of the different extensions are given schematically in Table 3 .

We observe that among these algebras, only those for which the condition $\left[K^{\prime}, W^{\prime}\right]=0$ is satisfied admit the subalgebra $\left\langle L_{i}^{\prime}, K_{i}^{\prime}, P_{i}^{\prime}, H^{\prime}\right\rangle \oplus\langle W\rangle$, the trilinear bracket of which being trivial. Actually this subalgebra can be considered as the cubic analogue of the static algebra of classical kinematics. Moreover, it is straightforward to verify that this subalgebra is always a contraction of the cubic algebra $\left\langle L_{i}^{\prime}, K_{i}^{\prime}, P_{i}^{\prime}, H^{\prime}\right\rangle \oplus\left\langle Q^{\prime}\right\rangle$ considered in fractional supersymmetry [20]:

$$
\left\{Q^{\prime}, Q^{\prime}, Q^{\prime}\right\}=3 H^{\prime}
$$


Table 3: Galilei algebras of order three

\begin{tabular}{|c||l|c|c|c|c|c|c|}
\hline & & {$\left[K^{\prime}, V^{\prime}\right]$} & {$\left[K^{\prime}, W^{\prime}\right]$} & $\left\{V^{\prime}, V^{\prime}, V^{\prime}\right\}$ & $\left\{V^{\prime}, V^{\prime}, W^{\prime}\right\}$ & $\left\{V^{\prime}, W^{\prime}, W^{\prime}\right\}$ & $\left\{W^{\prime}, W^{\prime}, W^{\prime}\right\}$ \\
\hline 1. & $a=\frac{1}{3}, b=\frac{4}{3}$ & $W^{\prime}$ & 0 & $P^{\prime}$ & 0 & 0 & 0 \\
\hline 2. & $1+a-b=0$ & $W^{\prime}$ & 0 & 0 & 0 & 0 & 0 \\
\hline 3. & $a=1, b=0$ & 0 & $V^{\prime}$ & 0 & 0 & $P^{\prime}$ & $H^{\prime}$ \\
\hline 4. & $1+b-a=0$ & 0 & $V^{\prime}$ & 0 & 0 & 0 & 0 \\
\hline 5. & $a=\frac{1}{3}$ & 0 & 0 & $P^{\prime}$ & 0 & 0 & 0 \\
\hline 6. & $a=b=\frac{1}{3}$ & 0 & 0 & $P^{\prime}$ & 0 & $P^{\prime}$ & 0 \\
\hline 7. & $a+2 b-1=0$ & 0 & 0 & 0 & 0 & $P^{\prime}$ & 0 \\
\hline
\end{tabular}

by simply considering the transformations

$$
W=\varepsilon Q
$$

Although it might appear surprising that (4.6) does not appear as a contraction of the cubic Poincare (or de Sitter) algebra, this is a direct consequence of the action of the Bose sector on the trilinear part and the bracket $\{W, W, W\}$. We will encounter this obstruction to recover this special extension also in later cases.

\section{Extensions of the Carroll algebra}

The Carroll algebra, first introduced in 2], is obtained from the Poincaré algebra through a contraction determined by rescaling the boosts and time translations:

$$
J_{i}^{\prime}=J_{i}, \quad K_{i}^{\prime}=\epsilon K_{i}, \quad P_{i}^{\prime}=P_{i}, \quad H^{\prime}=\epsilon H .
$$

The brackets are give on Table 1. Although appearing naturally in the classification of kinematical groups, as an alternative intermediate algebra in the contraction of the Poincaré group onto the static group, and therefore as another non-relativistic limit (the other being the Galilei algebra), the Carroll algebra has played no distinguished role in kinematics. However, recently it has been analysed whether this algebra constitutes an object in the study of tachyon condensates in string theory [21. It is in this context where a possible cosmological interpretation of this limit of the Poincare algebra and the supersymmetric extensions recover some interest. First, it is rather straightforward to construct a supersymmetric extension of the Carroll algebra, by adding to the generators specified in (5.1) the additional generators of the symmetric part

$$
Q_{\alpha}^{\prime}=\epsilon^{a} Q_{\alpha}, \quad \bar{Q}_{\alpha}^{\prime}=\epsilon^{b} \bar{Q}_{\alpha} .
$$

These transformations define a contraction if the constraint $a+b-1 \geq 0$ is satisfied. For the special case $a+b-1=0$ we get the $N=2$ supersymmetric extension of the Carroll algebra determined by

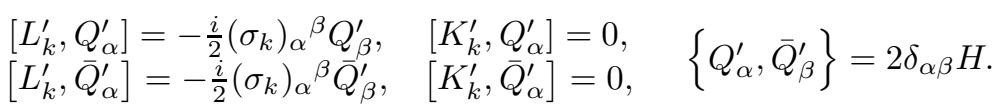

The remarkable fact of this extension is that in the Fermi sector the symmetric product reproduces a Clifford algebra structure. Indeed, this algebra corresponds to the $N=4$ supersymmetric quantum mechanics extension of the Galilei algebra. However, in contrast to the Galilei case, here the supercharges are in the spinor representation of the rotation group.

\subsection{Carroll algebras of order 3}

Following the same ansatz as before, we construct a Carroll algebra of order three adding new generators, in this case

$$
V_{i}^{\prime}=\epsilon^{a} V_{i}, \quad W^{\prime}=\epsilon^{b} W
$$


If we take the limit, this leads to the following constraints upon the parameters

$$
a \geq 0,2 a+b-1 \geq 0, a+2 b \geq 0, b \geq \frac{1}{3}, 1+b-a \geq 0,1+a-b \geq 0 .
$$

They have all to be satisfied in order to define a Lie of order three. It is not difficult to see that seven independent solutions to these conditions exist, given schematically in Table 4.

Table 4: Carroll algebras of order three

\begin{tabular}{|c||l|c|c|c|c|c|c|}
\hline & & {$\left[K^{\prime}, V^{\prime}\right]$} & {$\left[K^{\prime}, W^{\prime}\right]$} & $\left\{V^{\prime}, V^{\prime}, V^{\prime}\right\}$ & $\left\{V^{\prime}, V^{\prime}, W^{\prime}\right\}$ & $\left\{V^{\prime}, W^{\prime}, W^{\prime}\right\}$ & $\left\{W^{\prime}, W^{\prime}, W^{\prime}\right\}$ \\
\hline 1. & $a=0, b=1$ & $W^{\prime}$ & 0 & $P^{\prime}$ & $H^{\prime}$ & 0 & 0 \\
\hline 2. & $1+a-b=0$ & $W^{\prime}$ & 0 & 0 & 0 & 0 & 0 \\
\hline 3. & $a=\frac{4}{3}, b=\frac{1}{3}$ & 0 & $V^{\prime}$ & 0 & 0 & 0 & $H^{\prime}$ \\
\hline 4. & $1+b-a=0$ & 0 & $V^{\prime}$ & 0 & 0 & 0 & 0 \\
\hline 5. & $2 a+b-1=0$ & 0 & 0 & 0 & $H^{\prime}$ & 0 & 0 \\
\hline 6. & $a=b=\frac{1}{3}$ & 0 & 0 & 0 & $H^{\prime}$ & 0 & $H^{\prime}$ \\
\hline 7. & $b=\frac{1}{3}$ & 0 & 0 & 0 & 0 & 0 & $H^{\prime}$ \\
\hline
\end{tabular}

For all the cubic extensions the brackets $\left\{V^{\prime}, W^{\prime}, W^{\prime}\right\}$ vanishes. Among these solutions, the last is of special interest, since it admits the subalgebra $\left\langle L_{i}^{\prime}, K_{i}^{\prime}, P_{i}^{\prime}, H^{\prime}\right\rangle \oplus\left\langle W^{\prime}\right\rangle$ and corresponds to the fractional supersymmetry extending the Galilei algebra

$$
\left\{W^{\prime}, W^{\prime}, W^{\prime}\right\}=3 H^{\prime}
$$

\section{Extensions of the Newton algebra}

In contrast to the previous cases, the Newton-Hooke algebras are not contractions of the Poincaré algebra, but are obtained directly from the de Sitter algebras by speed-space contractions 8 determined by the transformations

$$
P_{i}^{\prime}=\epsilon P_{i}, \quad K_{i}^{\prime}=\epsilon K_{i} .
$$

The limit $\epsilon \rightarrow 0$ corresponds to the oscillating Newton Lie algebra. The various brackets are given on Table 1. The features of these models are therefore close to those of the de Sitter algebras, and have been studied in connection to certain properties of space-time curvature. Moreover, these algebras are known to appear as subalgebras of multi-temporal conformal algebras, and have been applied to non-relativistic branes 22 . The $N=2$ supersymmetric extension of the Newton algebra is obtained by joining to the generators of (6.1) the additional elements

$$
Q_{\alpha}=\epsilon^{a} S_{\alpha}, \quad \bar{Q}_{\alpha}=\epsilon^{b} \bar{S}_{\alpha}
$$

Performing the brackets it follows that the action of $P^{\prime}$ on $Q$ and $\bar{Q}$ is trivial, although the anti-commutators lead to the constraint $a+b-1 \geq 0$. If we take the equality $a+b-1=0$, which is the only possibility of obtaining a non-trivial symmetric bracket, we get

$$
\begin{array}{ll}
{\left[L_{k}^{\prime}, Q_{\alpha}^{\prime}\right]=-\frac{i}{2}\left(\sigma_{k}\right)_{\alpha}{ }^{\beta} Q_{\beta}^{\prime},} & {\left[K_{k}^{\prime}, Q_{\alpha}^{\prime}\right]=0, \quad\left\{Q_{\alpha}^{\prime}, \bar{Q}_{\beta}^{\prime}\right\}=2 \sigma_{i \alpha \beta} P^{\prime i}} \\
{\left[L_{k}^{\prime}, \bar{Q}_{\alpha}^{\prime}\right]=-\frac{i}{2}\left(\sigma_{k}\right)_{\alpha}{ }^{\beta} \bar{Q}_{\beta}^{\prime},} & {\left[K_{k}^{\prime}, \bar{Q}_{\alpha}^{\prime}\right]=0,}
\end{array}
$$

which provides the desired extension. We observe that supersymmetric extensions of the (oscillating) Newton algebras have already been considered in [5].

\footnotetext{
${ }^{8}$ These further have the Galilei algebra as non-relativistic limit. See Table 1.
} 


\subsection{Newton algebras of order 3}

To study the possible contractions of the $\mathfrak{s o}(2,3)$ - algebra of order 3 onto algebras of order 3 containing the Newton algebra in its bosonic part, we introduce the following notations that will facilitate the analysis:

$$
A_{M N}= \begin{cases}A_{k}=A_{i j}(i, j, k \text { perm. }), & B_{i}=A_{0 i} \\ C_{i}=A_{i 4}, & R=A_{04} .\end{cases}
$$

Over this basis we have

$$
\begin{array}{llll}
{\left[L_{i}, A_{j}\right]=A_{k}} & {\left[L_{i}, B_{j}\right]=B_{k}} & {\left[L_{i}, C_{j}\right]=C_{k}} & {\left[L_{i}, R\right]=0} \\
{\left[K_{i}, A_{j}\right]=B_{k}} & {\left[K_{i}, B_{j}\right]=-A_{k}} & {\left[K_{i}, C_{j}\right]=-\delta_{i j} R} & {\left[K_{i}, R\right]=-Q_{i}} \\
{\left[P_{i}, A_{j}\right]=C_{k}} & {\left[P_{i}, B_{j}\right]=\delta_{i j} R} & {\left[P_{i}, C_{j}\right]=-A_{k}} & {\left[P_{i}, R\right]=B_{i}} \\
{\left[H, A_{i}\right]=0} & {\left[H, B_{i}\right]=C_{i}} & {\left[H, C_{i}\right]=-B_{i}} & {[H, R]=0}
\end{array}
$$

and for the trilinear brackets

$$
\begin{aligned}
& \left\{A_{i}, A_{j}, A_{k}\right\}=-\delta_{i j} L_{k}-\delta_{i k} L_{j}-\delta_{j k} L_{i}, \\
& \left\{A_{i}, A_{j}, B_{k}\right\}=-\delta_{i j} K_{k}, \quad\left\{A_{i}, A_{j}, C_{k}\right\}=-\delta_{i j} P_{k}, \quad\left\{A_{i}, A_{j}, R\right\}=-\delta_{i j} H, \\
& \left\{A_{i}, B_{j}, B_{k}\right\}=-\delta_{j k} L_{i}, \quad\left\{A_{i}, C_{j}, C_{k}\right\}=-\delta_{j k} L_{i}, \quad\left\{A_{i}, R, R\right\}=L_{i}, \\
& \left\{A_{i}, C_{j}, B_{k}\right\}=0, \quad\left\{A_{i}, C_{j}, R\right\}=0, \quad\left\{A_{i}, B_{j}, R\right\}=0, \\
& \left\{B_{i}, B_{j}, B_{k}\right\}=-\delta_{i j} K_{k}-\delta_{i k} K_{j}-\delta_{j k} K_{i},\left\{B_{i}, B_{j}, C_{k}\right\}=-\delta_{i j} P_{k}, \\
& \left\{B_{i}, B_{j}, R\right\}=-\delta_{i j} H, \quad\left\{B_{i}, C_{j}, C_{k}\right\}=-\delta_{j k} K_{i}, \quad\left\{B_{i}, R, R\right\}=K_{i}, \quad\left\{B_{i}, C_{j}, R\right\}=0, \\
& \left\{C_{i}, C_{j}, C_{k}\right\}=-\delta_{i j} P_{k}-\delta_{i k} P_{j}-\delta_{j k} P_{i}, \quad \\
& \left\{C_{i}, C_{j}, R\right\}=-\delta_{i j} H, \quad\left\{C_{i}, R, R\right\}=P_{i}, \quad\{R, R, R\}=3 H .
\end{aligned}
$$

Performing the contraction defined by the scaling transformations

$$
A_{i}^{\prime}=\varepsilon^{a} A_{i}, B_{i}^{\prime}=\varepsilon^{b} B_{i}, C_{i}^{\prime}=\varepsilon^{c} C_{i}, R_{i}^{\prime}=\varepsilon^{d} R_{i},
$$

together with (6.1), the limit $\varepsilon \rightarrow 0$ gives rise to a consistent contraction pattern if the following constraints are satisfied: $b=c$ and the system of inequalities

$$
\begin{aligned}
& e_{1}=a \geq 0, e_{2}=2 a+b-1 \geq 0, e_{3}=2 a+d \geq 0, e_{4}=a+2 b \geq 0, e_{5}=a+2 d \geq 0, \\
& e_{6}=b-1 / 3 \geq 0, e_{7}=2 b+d \geq 0, e_{8}=b+2 d-1 \geq 0, e_{9}=d, e_{10}=1+a-b \geq 0, \\
& e_{11}=1+b-a \geq 0, e_{12}=1+b-d \geq 0, e_{13}=1+d-b \geq 0 .
\end{aligned}
$$

To simplify the presentation we write the non-zero brackets in matrix form as follows

$$
\mathbf{M}=\left(\begin{array}{cccc}
{\left[L^{\prime}, A^{\prime}\right]} & {\left[L^{\prime}, B^{\prime}\right]} & {\left[L^{\prime}, C^{\prime}\right]} & {\left[L^{\prime}, R^{\prime}\right]} \\
{\left[K^{\prime}, A^{\prime}\right]} & {\left[K^{\prime}, B^{\prime}\right]} & {\left[K^{\prime}, C^{\prime}\right]} & {\left[K^{\prime}, R^{\prime}\right]} \\
{\left[P^{\prime}, A^{\prime}\right]} & {\left[P^{\prime}, B^{\prime}\right]} & {\left[P^{\prime}, C^{\prime}\right]} & {\left[P^{\prime}, R^{\prime}\right]} \\
{\left[H^{\prime}, A^{\prime}\right]} & {\left[H^{\prime}, B^{\prime}\right]} & {\left[H^{\prime}, C^{\prime}\right]} & {\left[H^{\prime}, R^{\prime}\right]}
\end{array}\right)=\left(\begin{array}{cccc}
A^{\prime} & B^{\prime} & C^{\prime} & 0 \\
\varepsilon^{1+a-b} B^{\prime} & \varepsilon^{1+b-a} A^{\prime} & \varepsilon^{1+c-d} R^{\prime} & \varepsilon^{1+d-c} C^{\prime} \\
\varepsilon^{1+a-c} C^{\prime} & \varepsilon^{1+b-d} R^{\prime} & \varepsilon^{1+c-a} A^{\prime} & \varepsilon^{1+d-b} B^{\prime} \\
0 & \varepsilon^{b-c} C^{\prime} & \varepsilon^{c-b} B^{\prime} & 0
\end{array}\right)
$$

for the bilinear part

$$
\begin{aligned}
\mathbf{N} & =\left(\begin{array}{cccc}
\left\{A^{\prime}, A^{\prime}, A^{\prime}\right\} & \left\{A^{\prime}, A^{\prime}, B^{\prime}\right\} & \left\{A^{\prime}, A^{\prime}, C^{\prime}\right\} & \left\{A^{\prime}, A^{\prime}, R^{\prime}\right\} \\
\left\{A^{\prime}, B^{\prime}, B^{\prime}\right\} & \left\{A^{\prime}, C^{\prime}, C^{\prime}\right\} & \left\{A^{\prime}, R^{\prime}, R^{\prime}\right\} & \left\{B^{\prime}, B^{\prime}, B^{\prime}\right\} \\
\left\{B^{\prime}, B^{\prime}, C^{\prime}\right\} & \left\{B^{\prime}, B^{\prime}, R^{\prime}\right\} & \left\{B^{\prime}, C^{\prime}, C^{\prime}\right\} & \left\{B^{\prime}, R^{\prime}, R^{\prime}\right\} \\
\left\{C^{\prime}, C^{\prime}, C^{\prime}\right\} & \left\{C^{\prime}, C^{\prime}, R^{\prime}\right\} & \left\{C^{\prime}, R^{\prime}, R^{\prime}\right\} & \left\{R^{\prime}, R^{\prime}, R^{\prime}\right\}
\end{array}\right) \\
& =\left(\begin{array}{cccc}
\varepsilon^{3 a} L^{\prime} & \varepsilon^{2 a+b-1} K^{\prime} & \varepsilon^{2 a+c-1} P^{\prime} & \varepsilon^{2 a+d} H^{\prime} \\
\varepsilon^{a+2 b} L^{\prime} & \varepsilon^{a+2 c} L^{\prime} & \varepsilon^{a+2 d} L^{\prime} & \varepsilon^{3 b-1} K^{\prime} \\
\varepsilon^{2 b+c-1} P^{\prime} & \varepsilon^{2 b+d} H^{\prime} & \varepsilon^{b+2 c-1} K^{\prime} & \varepsilon^{b+2 d-1} K^{\prime} \\
\varepsilon^{3 c-1} P^{\prime} & \varepsilon^{2 c+d} H^{\prime} & \varepsilon^{c+2 d-1} P^{\prime} & \varepsilon^{3 d} H^{\prime}
\end{array}\right)
\end{aligned}
$$


for the trilinear part. Solving the system, we get 27 possible algebras. All the solutions have the following brackets in common: $\left[L^{\prime}, A^{\prime}\right]=A^{\prime},\left[L^{\prime}, B^{\prime}\right]=B^{\prime},\left[L^{\prime}, C^{\prime}\right]=C^{\prime},\left[H^{\prime}, B^{\prime}\right]=C^{\prime},\left[H^{\prime}, C^{\prime}\right]=B^{\prime}$. However, among these 27 algebras, only 19 are of real interest for providing non-trivial trilinear brackets. We therefore only list the solutions with non-vanishing ternary brackets. (The non-listed solutions correspond to the cases where the identity $e_{i}$ are indeed an equality for (1) $i=10,(2) i=11,(3) i=12$, (4) $i=13$, (5) $i=10,12$, (6) $i=10,13,(7) i=11,12,(8) i=11,13$ for which only some bilinear brackets are non-vanishing.) In all the solutions listed in Table 5 , since $\left[H^{\prime}, C^{\prime}\right]=B^{\prime}$, we do not have a solution analogous to fractional supersymmetric extension of the Galilei algebra (4.6) obtained from the Carroll algebra. This follows from the structure of the Newton algebras, where the time translation interchanges the space translations and the boosts, and is in perfect agreement with the fact that contracting the Poincaré algebra of order three does not lead to this exceptional cubic Galilean extension.

\subsection{Contractions of cubic Newton extensions}

Since the Newton algebra contracts onto the Galilei algebra by means of the transformations

$$
P^{\prime}=\varepsilon P, H^{\prime}=\varepsilon H
$$

it is expected that the transitivity of contractions implies that the cubic extensions of Newton contract onto some cubic extensions of the Galilei algebra (of dimension 20). It is straightforward to verify that choosing

$$
A^{\prime}=\varepsilon A, B^{\prime}=\varepsilon B, C^{\prime}=\varepsilon^{\frac{1}{3}} C, R^{\prime}=\varepsilon^{\frac{1}{3}} R,
$$

the trilinear brackets of the four elements $\left\{C_{i}, R\right\}$ is preserved. Further these transformations imply that, after the contraction, $P^{\prime}$ and $H^{\prime}$ commute with $A^{\prime}, B^{\prime}, C^{\prime}, R^{\prime}$. As a consequence, all the contractions admit a subalgebra spanned by the generators $\left\langle L_{i}, K_{i}, P_{i}, H>\oplus<C_{i}, R>\right.$, which therefore must correspond to some cubic extensions $E_{i}(i=1 . .7)$ of the Galilei algebra obtained in Table 3 . Denoting by $S_{j}$ the algebras of Table 5 , the preceding transformations give rise to the following contraction pattern:

1. $E_{1}$ arises from the contraction of $S_{9}, S_{11}$ and $S_{13}$.

2. $E_{2}$ arises from the contraction of $S_{16}$.

3. $E_{3}$ arises from the contraction of $S_{4}, S_{17}$ and $S_{18}$.

4. $E_{5}$ arises from the contraction of $S_{2}, S_{8}$ and $S_{14}$.

5. $E_{6}$ arises from the contraction of $S_{7}, S_{10}$ and $S_{12}$.

6. $E_{7}$ arises from the contraction of $S_{3}$ and $S_{15}$ and $S_{19}$.

Further, $S_{1} S_{5}$ and $S_{6}$ contract onto a trivial cubic extension of the Galilei algebra 9 . As for the algebra $E_{4}$, it follows from the contraction of the algebras $S_{4}, S_{17}$ and $S_{18}$ defined by the transformations:

$$
A^{\prime}=\varepsilon A, B^{\prime}=\varepsilon B, C^{\prime}=\varepsilon C, R^{\prime}=\varepsilon R
$$

in addition to those of (6.8). This shows that any of the Galilean algebras of order three can be obtained through two different directions, either starting from the extension of the Poincaré algebra (3.8), or considering the Newton algebras of order three. The remarkable fact is that in the latter case, the Galilean extensions are obtained as subalgebras of contractions, in contrast to the derivation developed in section 4. This points out the consistency of the contraction method, and justifies that no information was lost in considering mainly the algebra (3.8) and its contractions.

\footnotetext{
${ }^{9}$ That is, having zero bilinear and trilinear brackets.
} 
Table 5: Possible 3-algebras with Bose sector of Newton type and non-trivial ternary brackets.

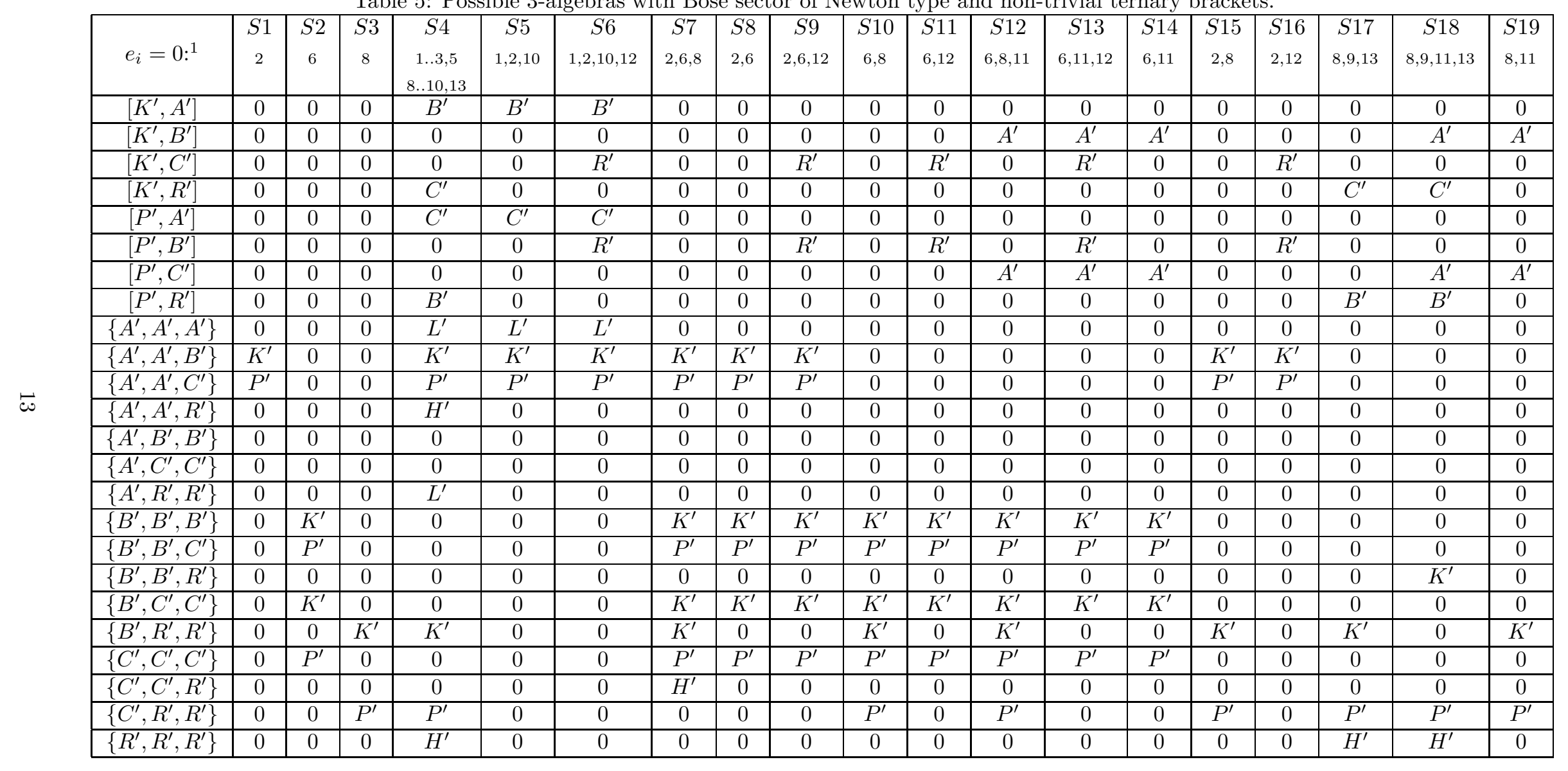

${ }^{a}$ For any solution $S$ the indices make reference to the inequalities of the system that are identities. For the non-appearing indices, the corresponding equations are strict inequalities 


\section{Conclusions}

Following the procedure undertaken by Bacry and Lévy-Leblond to classify the possible kinematics, we have extended the method, based on contractions of Lie algebras, to obtain a similar classification of "kinematical" superalgebras and Lie algebras of order three, basing on natural generalisations of the already known extensions that have been proven to be of physical interest. In most cases this is possible using the supersymmetric extension of the anti-de Sitter algebra. This contraction pattern gives rise to some of the Lie algebras of order three which have been used in physics with interesting applications (see e.g. [9]). However, some new cubic algebras emerge naturally is this classification scheme. Whether or not they could have some physical interesting interpretation is still an open question. In this paper, various extensions arising directly from the cubic de Sitter algebras have been left out, which provide other alternative models. However, due to their non-apparent relation with the established field theoretic realizations, they seem not to be very relevant from the physical point of view. The impossibility of deriving the Galilean extension considered in FSUSY and the supersymmetric Quantum Mechanics shows that some possibilities are lost when generalising the contraction procedure from Lie algebras to Lie algebras of order three. This exceptions are however expectable from the theory, and do not constitute a limitation of the method.

It should also be remarked that the classical kinematics are based on a heavy assumption, namely, that parity and time reversal (short PT) are automorphisms of the kinematical group. However, as follows from the theory of weak interactions, this hypothesis is physically objectionable. Moreover, it is known that a permanent neutron electric dipole moment requires parity and time-reversal violation [23], which invalidates partially the physical assumptions made in [1]. A classification of kinematics based only on spatial isotropy exists [24, and the resulting algebras are also related by contractions. However, these additional algebras

have no relation with the de Sitter algebras, that is, no contraction of the latter leads to these models without (PT). Moreover, they may also depend on parameters, which makes a physical interpretation of the generators quite difficult. Due to the non-existence of some relation between the non-classical kinematical algebras and semisimple Lie algebras, the possibility of obtaining supersymmetric and cubic extensions is probably very reduced, if feasible at all.

\section{Acknowledgement}

One of the authors (RCS) acknowledges partial financial support from the research project MTM2006-09152 of the Ministerio de Educación y Ciencia. The authors are indebted to J. A. de Azcárraga for useful comments and references [6].

\section{A Lie algebra of order $F$-definition}

We summarize here for completeness the definition of a (real) elementary Lie algebra of order three, since in this paper we are dealing with this type of algebras. More details can be found in 8 . The real vector space $\mathfrak{g}=\mathfrak{g}_{0} \oplus \mathfrak{g}_{1}$ is called an elementary Lie algebra of order three if

1. $\mathfrak{g}_{0}$ is a (real) Lie algebra.

2. $\mathfrak{g}_{1}$ is a (real) representation of $\mathfrak{g}_{0}$. If $X \in \mathfrak{g}_{0}, Y \in \mathfrak{g}_{1}$, then $[X, Y]$ denotes the action of $X \in \mathfrak{g}_{0}$ over $Y \in \mathfrak{g}_{1}$.

3. There exists a trilinear, $\mathfrak{g}_{0}$-equivariant map

$$
\{,,\}: \mathcal{S}^{3}\left(\mathfrak{g}_{1}\right) \rightarrow \mathfrak{g}_{0},
$$

where $\mathcal{S}^{3}\left(\mathfrak{g}_{1}\right)$ denotes the three-fold symmetric product of $\mathfrak{g}_{1}$.

4. For $Y_{1}, \cdots, Y_{4} \in \mathfrak{g}_{1}$ the following "Jacobi identity" holds:

$$
\left[Y_{1},\left\{Y_{2}, Y_{3}, Y_{4}\right\}\right]+\left[Y_{2},\left\{Y_{3}, Y_{4}, Y_{1}\right\}\right]+\left[Y_{3},\left\{Y_{4}, Y_{1}, Y_{2}\right\}\right]+\left[Y_{4},\left\{Y_{1}, Y_{2}, Y_{3}\right\}\right]=0 .
$$


It turns out that Lie algebras of order three appear as some kind of generalization of Lie algebras and superalgebras. In 8 the definition of (complex) Lie algebras of order $F$ (elementary or not) has been given. Indeed, in the complex case the situation is more rich, since there is, in addition to the real case, an automorphism which induces a grading of the (complex) vector space.

\section{References}

[1] H. Bacry and J. Lévy-Leblond, Possible kinematics, J. Math. Phys. 9 (1968) 1605-1614; J. F. Cariñena, M. A. del Olmo and M. Santander, Kinematic groups and dimensional analysis, J. Phys. A: Math. Gen. 14 (1981) 1-14.

[2] J. M. Lévy-Leblond, Une nouvelle limite non-relativiste du groupe de Poincaré, Ann. Inst. H. Poincaré serie A 3 (1965), 1-12.

[3] R. Kerner, Non-commutative Geometry, in Mathematical Physics towards the XXI Century, R. N. Sen and A. Gersten, eds., Beersheva 1993, 112-129.

[4] M. de Montigny, J. Niederle, and A. P. Nikitin, Galilei invariant theories. I. Constructions of indecomposable finite-dimensional representations of the homogeneous Galilei group: directly and via contractions, J. Phys. A: Math. gen. 39 (2006), 9365-9385. M. de Montigny, Contractions and deformations of Lie algebras, in Quantum theory and symmetries (World Sci. Publ., Hackensack, NJ, 2004), 65-70.

[5] V. Hussin, J. Negro and M. A. del Olmo, Kinematical superalgebras, J. Phys. A: Math. Gen. 32 (1999) 5097-5121. A. Ballesteros, F. J. Herranz and J. Negro, Boson representations, non-standard quantum algebras and contractions, J. Phys. A: Math. Gen. 30 (1997), 6797-6809.

[6] R. Puzalowski, Galilean supersymmetry, Acta Phys. Austriaca 50 (1978), 45-52. J.A. de Azcarraga, D. Ginestar, Nonrelativistic limit of supersymmetric theories, J.Math.Phys. 32 (1991), 3500-3508.

[7] J. M. Lévy-Leblond, Galilei group and Galilean invariance, in Group Theory and Applications, ed E. M. Loebl (Academic Press, New-York, 1971), 221-299.

[8] M. Rausch de Traubenberg, M. J. Slupinski, Fractional supersymmetry and F(th) roots of representations, J. Math. Phys. 41 (2000), 4556-4579, arXiv:hep-th/9904126]. M. Rausch de Traubenberg, M. J. Slupinski, Finite-dimensional Lie algebras of order F, J. Math. Phys. 43 (2002), 5145-5160, arXiv:hep-th/0205113.

[9] N. Mohammedi, G. Moultaka and M. Rausch de Traubenberg, Field theoretic realizations for cubic supersymmetry, Int. J. Mod. Phys. A 19, 5585 (2004), arXiv:hep-th/0305172. G. Moultaka, M. Rausch de Traubenberg and A. Tanasa, Cubic supersymmetry and abelian gauge invariance, Int. J. Mod. Phys.A20, 5779 (2005) hep-th/0411198.

[10] M. Goze, M. Rausch de Traubenberg and A. Tanasa, Poincaré and $\mathfrak{s l}(2)$ algebras of order 3, J. Math. Phys. 48 (2007) 093507 arXiv:math-ph/0603008.

[11] I. E. Segal, A class of operator algebras which are determined by groups, Duke. Math. J. 18 (1951), 221265. E. Inönü and E. P. Wigner, On the Contraction of Groups and Their Representations, Proc. Nat. Acad. Sci. 39 (1953), 510-521.

[12] Ya. Kh. Lykhmus, The simplest contractions of nonassociative algebras, Eesti NSV Tead. Akad. Toimetised Fü̈s.-Mat. 20 (1971), 213-215.

[13] R. Campoamor-Stursberg, Internal labelling operators and contractions of Lie algebras, J. Phys. A: Math. Theor. 40 (2007), 14773-14791 arXiv:hep-th/0706.2581.

[14] M. Rausch de Traubenberg, Clifford algebras in physics, to appear in Proceedings of the 7th International Conference on Clifford algebras and their applications. (ICCA), May 2005, Toulouse, arXiv:hep-th/0506011. 
[15] J. Wess, J. Bagger, Supersymmetry and Supergravity (Princeton, USA: Univ. Pr. (1992) 259 p)

[16] P. C. West, Introduction to supersymmetry and supergravity (Singapore, Singapore: World Scientific (1990) $425 \mathrm{p}$ ).

[17] P. G. O. Freund, I. Kaplansky, Simple Supersymmetries, J. Math. Phys. 17 (1976), 228-231. W. Nahm, Supersymmetries and their Representations, Nucl. Phys. B135 (1978), 149-166.

[18] Yu. A. Gol'fand and E. P. Likhtman, Extension of the Algebra of Poincaré Group Generators and Violation of P-Invariance, JETP Letters 13 (1971), 452-455; J. Wess and B. Zumino, A Lagrangian Model Invariant by Supergauge Transformations, Phys. Lett. B49 (1974), 52-54.

[19] B. G. Konopel'chenko, On an extension of the Poincaré algebra with spinorial generators, JETP Letters 21 (1975), 612-614.

[20] J. L. Matheus-Valle and Marco A. R. Monteiro, Quantum group generalization of the classical supersymmetric point particle, Mod. Phys. Lett. A7 (1992) 3023; S. Durand, Extended fractional supersymmetric quantum mechanics, Mod. Phys. Lett A8 (1993) 1795, hep-th/9305130 J. A. de Azcàrraga and A. J. Macfarlane, Group theoretical foundations of fractional supersymmetry, J. Math .Phys. 37 (1996) 1115, hep-th/9506177. N. Fleury and M. Rausch de Traubenberg, Local Fractional Supersymmetry for Alternative Statistics, Mod. Phys. Lett. A11 (1996) 899, hep-th/9510108, R. S. Dunne, A. J. Macfarlane, J. A. de Azcarraga and J. C. Perez Bueno, Geometrical foundations of fractional supersymmetry, Int. J. Mod. Phys. A 12 (1997) 3275, hep-th/9610087.

[21] G. Gibbons, K. Hashimoto and P. Yi, Tachyon condensates, carrollian contraction of Lorentz group, and fundamental strings, JHEP09 (2002), 061.

[22] F. J. Herranz, M. Santander, A new Lie algebra expansion method: Galilei expansions to Poincare and Newton-Hooke, math-ph/9909005. J. Brugueś, J. Gomis and K. Kamimura, Newton-Hooke algebras, nonrelativistic branes, and generalized pp-wave metrics, Phys. Rev. D73, 085011 (2006).

[23] P. Herczeg, in Parity and Time-Reversal Violation in Compound Nuclear States and Related Topics, eds. N. Auerbach and J. Bowman (World Scientific, Singapore 1996), p 214. P. G. H. Sandars, Parity and Time-Reversal Violation in Atoms and Molecules, Physica Scripta 36 (1987), 904-910.

[24] H. Bacry and J. Nuyts, Classification of Ten Dimensional Kinematical Groups with Space Isotropy, J. Math .Phys. 27 (1986) 2455-2457. 